\title{
Local Effects of Two Intravenous Formulations of Pulmonary Vasodilators on Airway Epithelium
}

\author{
Bradley A Kuch, Rosalie Linssen, Hiroki Yoshikawa, Craig D Smallwood, ${ }^{\dagger}$ and Michael D Davis
}

\begin{abstract}
BACKGROUND: Intravenous formulations of epoprostenol are frequently delivered via nebulizer to treat pulmonary hypertension in acutely ill patients. Although their efficacy as pulmonary vasodilators has been shown to be comparable to inhaled nitric oxide, the local effects of these formulations within the airways have not been determined. We hypothesized that the alkaline diluents of these compounds would lead to increased airway epithelial cell death and ciliary cessation. METHODS: Human bronchial epithelial cells were exposed to epoprostenol in glycine and arginine diluents or control fluid. Ciliary beat frequency, lactate dehydrogenase, and total RNA levels were measured before and after exposure. Results were compared between exposure and control groups. RESULTS: Ciliary beat frequency ceased immediately after exposure to epoprostenol with both diluents. Lactate dehydrogenase levels increased by $200 \%$ after exposure to epoprostenol and glycine diluent $(P=.002)$. Total RNA levels were undetectable after exposure to epoprostenol and arginine, indicating complete cell death and lysis $(P=.015)$. Ciliary beat frequency ceased after $30 \mathrm{~s}$ of exposure to epoprostenol and glycine $(P=\mathbf{. 0 0 8})$. There was no difference between cells exposed to epoprostenol and those exposed only to diluent. CONCLUSIONS: Exposure to intravenous formulations of epoprostenol in glycine and arginine caused increased cell death and ciliary cessation in bronchial epithelial cells. These findings suggest that undesired local effects may occur when these compounds are delivered as inhaled aerosols to patients. Key words: airway $\mathrm{pH}$; inhaled epoprostenol; inhaled prostacyclin; pulmonary vasodilator. [Respir Care 2020;65(10):1427-1432. (C) 2020 Daedalus Enterprises]
\end{abstract}

\section{Introduction}

Inhaled nitric oxide (INO) and continuously nebulized inhaled epoprostenol have been used to treat pulmonary hypertension in neonatal, pediatric, and adult populations. ${ }^{1,2}$ INO and epoprostenol exhibit similar effectiveness in increasing oxygenation, decreasing pulmonary artery pressure, and decreasing right-ventricular afterload in ARDS. ${ }^{3-7}$ These

\footnotetext{
Mr Kuch is affiliated with the Department of Pediatric Critical Care Medicine and Respiratory Care Services, UPMC Children's Hospital of Pittsburgh, Pittsburgh, Pennsylvania. Drs Linssen and Yoshikawa are affiliated with the Division of Pulmonary Medicine, Children's Hospital of Richmond at Virginia Commonwealth University, Richmond, Virginia. Dr Smallwood was affiliated with the Division of Critical Care Medicine, Department of Anesthesiology, Critical Care and Pain Medicine, Boston Children's Hospital, Boston, Massachusetts and the Harvard Medical School, Boston, Massachusetts. Dr Davis is affiliated with the Wells Center for Pediatric Research/Pulmonology, Allergy, and Sleep Medicine, Riley Hospital for Children at Indiana University, Indianapolis, Indiana.
}

$\dagger$ Deceased. effects have also been observed in patients who underwent cardiothoracic surgery. ${ }^{8-10}$ In patients receiving heart or lung transplants, INO and epoprostenol are thought to decrease reperfusion injury while acting as a selective pulmonary vasodilator, improving hemodynamic indexes. ${ }^{11}$

Currently, INO is the most commonly used, FDAapproved inhaled pulmonary vasodilator, and it is approved for use in neonates ( $>34$ weeks gestation) with hypoxic respiratory failure and associated pulmonary hypertension. ${ }^{12}$ Administration of INO outside of this indication is offlabel but common across all age groups. Increasing

\footnotetext{
Dr Davis is supported in part by an investigator-initiated research award from Mallinckrodt Pharmaceuticals and by NIH P01HL128192. Mr Kuch has disclosed relationships with Smiths Medical and Mallinckrodt Pharmaceuticals. The other authors have disclosed no conflicts of interest.

Correspondence: Michael D Davis RRT PhD FAARC, Wells Center for Pediatric Research, Pulmonology, Allergy, and Sleep Medicine, Riley Hospital for Children at Indiana University. E-mail: mdd1@iu.edu.
}

DOI: $10.4187 /$ respcare. 07938 
health care costs coupled with declining reimbursements rates have led to efforts to decrease direct-delivery costs of INO. Continuously nebulized inhaled epoprostenol

\section{SeE the Related Editorial on Page 1626}

may be a cost-effective alternative to INO; the cost of INO is approximately $\$ 220.46 / \mathrm{h}$ compared to an hourly cost of \$2.01-\$10.05 for inhaled epoprostenol for a 70$\mathrm{kg}$ adult patient. ${ }^{2,13}$ These potential cost savings have led to interest in substituting inhaled epoprostenol in patients who would otherwise receive INO. ${ }^{10}$

Although there is evidence that epoprostenol offers clinical benefits similar to those of INO, safety data are very limited. Significant safety concerns remain regarding the continuous nebulization of epoprostenol, including ventilator malfunction, unrecognized nebulizer failure, and a lack of mandated back-up delivery systems. ${ }^{1,2}$ Further, preparations of epoprostenol with either glycine or sucrose/L-arginine buffers have exceedingly high $\mathrm{pH}$ levels of 11-13, which may have negative effects on airway epithelia including increased inflammation, ciliary dysfunction, decreased transport of bronchodilators, and cell death. ${ }^{1,14-19}$ An independent thirdparty report highlighted potential technical errors, which include infusion pump misconnections, over- or underdosing, a lack of alarms on nebulizers to identify stoppage of dose delivery, and ventilator component malfunction leading to auto-PEEP and pneumothorax. ${ }^{20}$

Given the alkaline properties of epoprostenol and its unknown effects on airway epithelium, we sought to evaluate the effects of glycine-buffered epoprostenol (EPO-GLY) and sucrose/L-arginine-buffered epoprostenol (EPO-ARG) on inflammatory biomarkers in differentiated human airway cells. Our group previously reported that human airway epithelial cells can be grown in an air-liquid interface that allows them to differentiate fully into functioning segments of airway epithelium. $^{21,22}$ This model allows for a controlled evaluation of airway epithelial function and response without systemic artifact. We hypothesized that exposure to EPO-GLY and EPO-ARG would increase levels of lactate dehydrogenase (LDH), a validated marker of cell stress and death, and that the effects seen in the epoprostenol groups will be caused by the alkaline GLY and ARG diluents as opposed to the epoprostenol. ${ }^{23,24}$ We further hypothesized that exposure to these compounds would decrease airway epithelial ciliary beat frequency.

\section{Methods}

Primary normal human bronchial epithelial cells used for all experiments described were from a healthy, adult, nonsmoker donor (Lifeline Cell TechnologyConnecticut). We used the BronchiaLife Basal Media (Lifeline Cell Technology, Frederick, Maryland) during cell growth, and

\section{QUICK LOOK}

\section{Current knowledge}

Intravenous formulations of epoprostenol are routinely delivered via nebulizer to acutely ill patients and are effective as pulmonary vasodilators. Concerns exist regarding their safe delivery and local effects within the airways.

\section{What this paper contributes to our knowledge}

Exposure of intravenous formulations of epoprostenol that are commonly delivered by nebulizer to the airway epithelium caused increased cell death and decreased ciliary beat frequency when tested in vitro. These effects were due to the high $\mathrm{pH}$ of the intravenous formulation rather than the epoprostenol itself.

we used homemade differentiation media, as described previously, during cell differentiation. ${ }^{21,22}$ Cells were differentiated on 6-mm Corning Transwells (Corning, New York) at 200,000 cells per well as previously described by our group and others. ${ }^{21,22}$ Differentiation as pseudostratified, ciliated, columnar epithelial cells was verified with direct visualization of ciliary movement with a $20 \times$ microscope.

LDH protein levels were evaluated in culture media using commercially available Pierce LDH cytotoxicity colorimetric assay kits (Thermo Fisher Scientific, Waltham, Massachusetts). Assays were run according to the manufacturer's specifications in undiluted media. Total RNA levels were evaluated in airway exposure fluids using a NanoDrop 8000 UV-Vis Spectrophotometer (Thermo Fisher Scientific) as previously described by our group. ${ }^{21}$

Under $20 \times$ magnification with a microscope, ciliary motion was directly visualized uniformly across all of the cell culture. We also recorded ciliary motion on video using an iPhone SE (Apple, Cupertino, California) mounted on a Gosky Universal Adapter Mount (GOSKY Optics, Zhejiang, China) after locking focus and exposure.

Each video was transformed to grayscale $(0=$ black, $1=$ white). Regions of interest were identified for each video by recording the coordinates of clusters of cells that demonstrated motion, and these regions were used for subsequent ciliary beat frequency analysis. Preference for each region of interest was given to cells where the cell wall was most clearly visible and not obscured by other cells. Next, individual regions were plotted (Fig. 1), and the number of peaks was calculated over the measurement epoch. The frequency was determined by adding the number of peaks and dividing by the sampling time provided in the video $(15 \mathrm{~s}$ for each video). In the cases where no cell motion could be detected by the software, this was noted in the study results.

After epithelial segments were fully differentiated and ciliary motion was confirmed, the Transwells were divided 

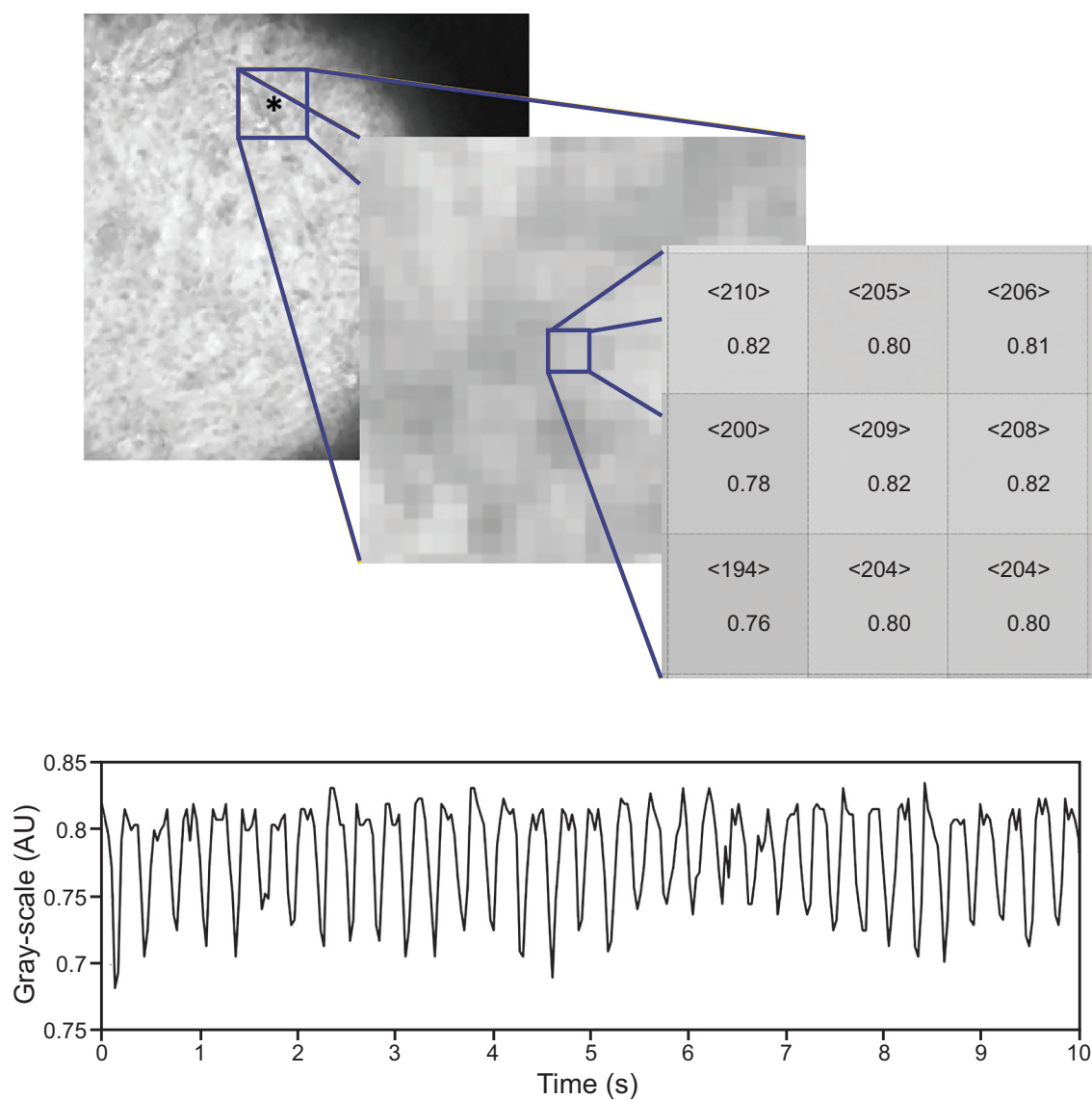

Fig. 1. Example of the calculation of ciliary beat frequency. First, a region of interest is manually recorded based on observed cell motion (indicated by *). The motion of the cells is analyzed by plotting the grayscale intensity over time. Ciliary beat frequency is calculated by adding the number of peaks detected and dividing by the total time of the recording.

equally into control and exposure groups. The $\mathrm{pH}$ of all exposure fluids was measured with a standard Mettler Toledo $\mathrm{pH}$ probe immediately prior to exposure and were confirmed to be 7 for the phosphate-buffered saline (Wet Control), 12 for the EPO-GLY and GLY, and 12.9 for the EPO-ARG and ARG, as indicated on their package inserts. All groups other than the Dry Control group were exposed to $40 \mu \mathrm{L}$ of sterile phosphate-buffered saline (ie, the minimum volume that uniformly coated the airway segments) to allow for uniform ciliary beat visualization. Video was recorded prior to exposure to establish a baseline. Following baseline establishment, $10 \mu \mathrm{L}$ of Wet Control, GLY, ARG, EPO-GLY, or EPO-ARG was added to each group. Then epoprostenol $(30,000 \mathrm{ng} / \mathrm{mL}$ concentration) was used in both epoprostenol groups because this concentration is commonly used in vivo. A Dry Control group was included in the protein analysis to determine any cell stress caused solely by exposure to fluid. Cells were visualized throughout the exposure with $20 \times$ magnification, and video was recorded. After 2 min of exposure, exposure fluids and cell culture media were removed and stored at $-80^{\circ} \mathrm{C}$ until LDH and total RNA measurement occurred.

\section{Statistical Analysis}

Sample sizes of 4 per group were determined to be appropriate to achieve a level of significance of .05 and a power of 0.80 with an effect size of 0.9 (G*Power Software, Kiel University, Kiel, Germany). Statistical analysis was performed using SigmaStat (Systat Software, San Jose, California). The unpaired Student 2-tailed $t$ test was used to compare LDH protein levels in control groups and stimulation groups. The Wilcoxon rank-sum test was used for non-Gaussian distributed data as determined with the Shapiro-Wilk test. The Wilcoxon matched-pairs signed rank test was used to assess differences in ciliary beat frequency between the control group and the treated group. A $P$ value of $<.05$ was considered statistically significant. A Bonferroni correction was used to account for multiplicity.

\section{Results}

LDH levels in groups exposed to EPO-GLY and GLY were $>200 \%$ higher than the levels in the Wet Control group and the Dry Control group (Fig. $2 \mathrm{~A}, P=.002$ ). Exposure to 

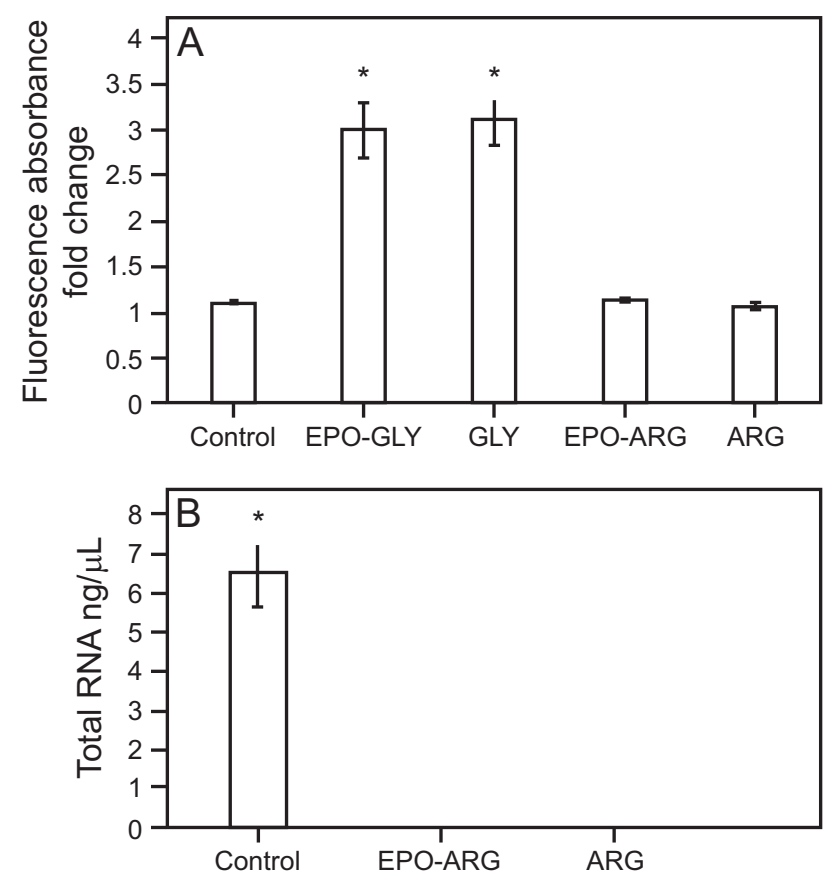

Fig. 2. A: Cells exposed to glycine-buffered epoprostenol (EPO-GLY) and glycine (GLY) released $200 \%$ more lactate dehydrogenase than the control group. No lactate dehydrogenase was measured from cells exposed to sucrose/L-arginine-buffered epoprostenol (EPO-ARG) or arginine (ARG). B: No RNA is detectable in the EPO-ARG and ARG cells after exposure, indicating complete death and lysis of cells. ${ }^{*} P<.05$.

EPO-ARG and ARG did not increase LDH levels compared to either control group, although ciliary cessation and cellular debris were noted within $30 \mathrm{~s}$ of exposure to these compounds. The exposure fluids of these groups were therefore analyzed for total RNA to determine if cell death occurred uniformly. Total RNA levels were undetectable in EPO-ARG and ARG exposure groups compared to control groups, indicating complete cell death and lysis (Fig. $2 \mathrm{~B}, P=.02$ ).

After $30 \mathrm{~s}$ of exposure to EPO-GLY, EPO-ARG, GLY, and ARG, all ciliary motion ceased by direct visualization. When evaluated using digital video analysis, the GLY exposure resulted in a reduction in mean ciliary beat frequency from $4.7 \pm 1.3 \mathrm{~Hz}$ to $0 \pm 0 \mathrm{~Hz}$ in control (Fig. 3, $P=.008$ ). Although ciliary motion was confirmed visually, the frequency analysis on the ARG exposure group could not be performed due to insufficient number of cilia detected by the software in these exposure videos.

\section{Discussion}

In this in vitro study, we describe the effects of 2 preparations of epoprostenol and their diluents on ciliary beat frequency and cell viability in healthy human bronchial epithelial cells. Apical exposure of pseudostratified, ciliated, columnar epithelium to epoprostenol simulated the

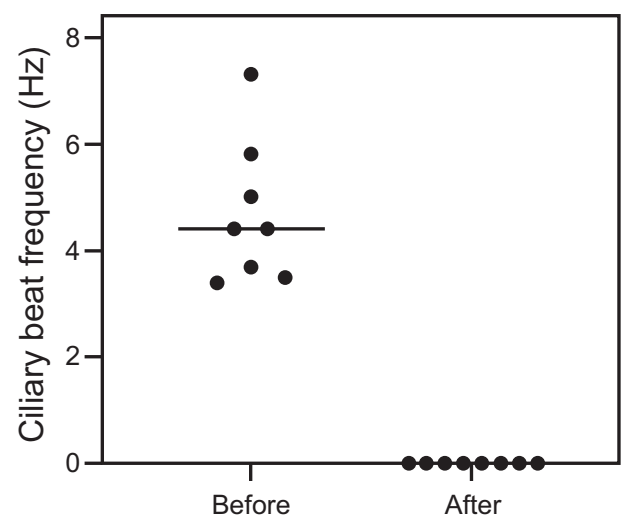

Fig. 3. Ciliary beat frequency before and after exposure to glycinebuffered epoprostenol (EPO-GLY). Ciliary beat frequency decreased to zero after exposure to EPO-GLY. $P=.008$.

conditions of airway exposure to these compounds from continuous aerosol delivery during invasive mechanical ventilation. Ciliary cell exposure to EPO-GLY, EPO-ARG, GLY, or ARG resulted in a statistically significant reduction in ciliary beat frequency and increased cell death compared to control cultures. There was no difference in outcomes between EPO-GLY and GLY or between EPO-ARG and ARG, suggesting that these results are caused by the ARG and GLY diluents rather than by the epoprostenol itself, although this cannot be completely ruled out. To our knowledge, this is the first study to demonstrate the direct effect of these compounds on airway cells.

Drug delivery can be inconsistent. The efficacy of any aerosolized medication is highly variable due to the efficiency of different nebulizers, physicochemical properties of different medications, and the various aerosol-generation technologies (ie, ultrasonic, vibrating mesh, and jet). ${ }^{25,26}$ Nebulizers generate a range of particle sizes that have a direct effect on the effectiveness of the aerosolized medication. $^{27,28}$ Particles $>5 \mu \mathrm{m}$ in diameter generated by the nebulizer are more likely to be deposited in the ventilator circuit and large airways. ${ }^{29,30}$ Importantly, for nebulized medications that have a desired mechanism of action at the level of the alveolar-capillary membrane (eg, pulmonary vasodilators), deposition in large airways offers limited or no clinical benefit and carries a potential increased risk of adverse effects. Further, variability in nebulized particle size and the resultant changes in distribution of drug deposition lead to clinically important differences in physiologic response. ${ }^{1}$ However, further work in this area is required.

Previous studies have noted unwanted side effects such as a decrease in systolic blood pressure requiring clinical intervention in up to $30 \%$ of pediatric subjects receiving aerosolized epoprostenol. ${ }^{31}$ However, the hemodynamic profile of adult subjects receiving aerosolized epoprostenol has been shown to be stable. This may be due to differences in volume distribution, ratio of surface area to body mass, 
and differences in absorption kinetics of the neonatal and pediatric populations compared to adults. ${ }^{8,9,11,32}$

The high $\mathrm{pH}$ of the diluents for epoprostenol may have undesirable effects on airway epithelium. ${ }^{1,14-19}$ Large particles (ie, $\geq 9.5 \mu \mathrm{m}$ in diameter) are reported to have an increased rate of deposition in the trachea. ${ }^{29}$ This suggests that the proportion of aerosolized particles of large size would have an increased probability of collecting in the trachea and large conducting airways where ciliary function is essential. Poor nebulizer function and changes in aerosol output may increase heterogeneous deposition of medications in the respiratory tract and increase the proportion of these droplets. Of note, a single 2.5-mL nebulization of a glycine buffer formulated for inhalation and buffered to a $\mathrm{pH}$ of 9.8 is sufficient to alter airway $\mathrm{pH}^{33}$ Our findings suggest that continuous exposure to these aerosolized diluents may result in airway cell death and decreased mucociliary clearance, but clinical studies are needed to assess this possibility.

This is not the first report of therapeutic agents that have the potential to injure the airways when delivered as aerosols. ${ }^{34}$ Unknown risks should be assumed when intravenous drug formulations are used for inhalational administration because the topical effects of these formulations on airway epithelium may not be known. We found a $200 \%$ increase in LDH levels in cells exposed to EPO-GLY and GLY and complete cell death according to RNA quantification in those exposed to EPOARG and ARG. These results suggest that the direct effect of high-pH solution on airway tissue results in cell death, although other mechanisms of cell death cannot be completely ruled out. We also observed complete cessation of ciliary beat frequency after exposure to these compounds. Mucociliary function is important in patients recovering from airway infection or respiratory failure. Decreasing mucociliary clearance and increasing cell death may worsen pulmonary system function; patients with ARDS or other critical pulmonary illness, who are often the recipients of inhaled vasodilators, may be even more susceptible to the effects we observed. ${ }^{35}$

In addition to the direct effects of epoprostenol and its diluents demonstrated in this study, patient safety concerns have been raised concerning the continuous nebulization of epoprostenol, such as technical sources of error, adverse effects, and physiologic deterioration..$^{31,36,37}$ Reported sources of technical error include the misconnection of infusion pumps, which can lead to over- or underdosing; nebulizers that lack alarms to alert the clinician of therapy interruption, which can result in physiologic deterioration; and ventilator malfunction from increased expiratory resistance, which limits exhalation and may result in pneumothorax. ${ }^{2,20}$ Technical risk factors need to be further explored to develop processes to decrease the likelihood of adverse events.

This study had several limitations. As with all in vitro studies, the findings may be subclinical or not representative of clinical effect. However, these results suggest that consideration of unwanted effects of aerosolized epoprostenol with
high-pH diluents is important. To control a uniform amount of drug exposure in each group, we elected to introduce the epoprostenol and diluents to the airway epithelia by volumetric pipette instead of via a nebulizer. This likely simulates the deposition, rainout, and collection of aerosolized medication in the larger airways more accurately than the entire delivery of aerosolized compounds to the targeted lower airways. In the future, it may be helpful to design an experiment that includes nebulization as a mechanism of introducing the agents. In addition, the method of ciliary motion measurement by digital video file analysis requires further optimization. Although ciliary motion was verified with direct visualization, the video files were unable to capture this movement in the ARG group. Higher-resolution cameras may make this technique more robust.

More work is needed to better understand the clinical effect that high-pH preparations have on airway tissue and relevant clinical outcomes. There is some debate in the literature as to the most effective aerosolized pulmonary vasodilator. It is recommended that nebulization of prostacyclin analogs approved and available in inhalational preparations should be considered for administration via the respiratory tract as a first-line aerosolized therapy for pulmonary hypertension or hypoxic respiratory failure. ${ }^{1,2}$ Agents such as iloprost, which is pharmacologically similar to epoprostenol with lower viscosity, greater stability, a longer high-life, and more physiologic $\mathrm{pH}$ may be a more suitable consideration. ${ }^{38}$

\section{Conclusions}

To our knowledge, this is the first report of airway epithelium exposure to EPO-GLY and EPO-ARG. This exposure resulted in rapid cell death and cessation of ciliary movement in vitro. These results were observed even in the absence of epoprostenol, suggesting they are likely due to the high $\mathrm{pH}$ of the GLY and ARG diluents. Future clinical studies are indicated to assess the clinical importance of these findings.

\section{REFERENCES}

1. Davis MD, Donn SM, Ward RM. Administration of inhaled pulmonary vasodilators to the mechanically ventilated neonatal patient. Paediatr Drugs 2017;19(3):183-192.

2. Kuch BA, Saville AL, Sanchez De Toledo J, Venkataraman ST. Inhaled pulmonary vasodilators: are there indications within the pediatric ICU? Respir Care 2017;62(6):678-698.

3. Domenighetti G, Stricker H, Waldispuehl B. Nebulized prostacyclin (PGI2) in acute respiratory distress syndrome: impact of primary (pulmonary injury) and secondary (extrapulmonary injury) disease on gas exchange response. Crit Care Med 2001;29(1):57-62.

4. Dunkley KA, Louzon PR, Lee J, Vu S. Efficacy, safety, and medication errors associated with the use of inhaled epoprostenol for adults with acute respiratory distress syndrome: a pilot study. Ann Pharmacother 2013;47(6):790-796.

5. van Heerden PV, Barden A, Michalopoulos N, Bulsara MK, Roberts BL. Dose-response to inhaled aerosolized prostacyclin for hypoxemia due to ARDS. Chest 2000;117(3):819-827. 


\section{Airway EfFects of PUlmonary Vasodilators}

6. Walmrath D, Schneider T, Schermuly R, Olschewski H, Grimminger F, Seeger W. Direct comparison of inhaled nitric oxide and aerosolized prostacyclin in acute respiratory distress syndrome. Am J Respir Crit Care Med 1996;153(3):991-996.

7. Zwissler B, Kemming G, Habler O, Kleen M, Merkel M, Haller M, et al. Inhaled prostacyclin (PGI2) versus inhaled nitric oxide in adult respiratory distress syndrome. Am J Respir Crit Care Med 1996;154(6 Pt 1):16711677.

8. De Wet CJ, Affleck DG, Jacobsohn E, Avidan MS, Tymkew H, Hill LL, et al. Inhaled prostacyclin is safe, effective, and affordable in patients with pulmonary hypertension, right heart dysfunction, and refractory hypoxemia after cardiothoracic surgery. J Thorac Cardiovasc Surg 2004;127(4):1058-1067.

9. McGinn K, Reichert M. A comparison of inhaled nitric oxide versus inhaled epoprostenol for acute pulmonary hypertension following cardiac surgery. Ann Pharmacother 2016;50(1):22-26.

10. Rao V, Ghadimi K, Keeyapaj W, Parsons CA, Cheung AT. Inhaled nitric oxide (iNO) and inhaled epoprostenol (iPGI2) use in cardiothoracic surgical patients: is there sufficient evidence for evidence-based recommendations? J Cardiothorac Vasc Anesth 2018;32(3):1452-1457.

11. Khan TA, Schnickel G, Ross D, Bastani S, Laks H, Esmailian F, et al. A prospective, randomized, crossover pilot study of inhaled nitric oxide versus inhaled prostacyclin in heart transplant and lung transplant recipients. J Thorac Cardiovasc Surg 2009;138(6):1417-1424.

12. DiBlasi RM, Myers TR, Hess DR. Evidence-based clinical practice guideline: inhaled nitric oxide for neonates with acute hypoxic respiratory failure. Respir Care 2010;55(12):1717-1745.

13. Pierce CM, Peters MJ, Cohen G, Goldman AP, Petros AJ. Cost of nitric oxide is exorbitant. BMJ 2002;325(7359):336.

14. Cancado JE, Mendes ES, Arana J, Horvath G, Monzon ME, Salathe M, Wanner A. Effect of airway acidosis and alkalosis on airway vascular smooth muscle responsiveness to albuterol. BMC Pharmacol Toxicol 2015;16:9.

15. Clary-Meinesz C, Mouroux J, Cosson J, Huitorel P, Blaive B. Influence of external $\mathrm{pH}$ on ciliary beat frequency in human bronchi and bronchioles. Eur Respir J 1998;11(2):330-333.

16. Holma B, Hegg PO. pH- and protein-dependent buffer capacity and viscosity of respiratory mucus. Their interrelationships and influence on health. Sci Total Environ 1989;84:71-82.

17. Holma B, Lindegren M, Andersen JM. pH effects on ciliomotility and morphology of respiratory mucosa. Arch Environ Health 1977;32 (5):216-226

18. Horvath G, Schmid N, Fragoso MA, Schmid A, Conner GE, Salathe $\mathrm{M}$, Wanner A. Epithelial organic cation transporters ensure $\mathrm{pH}$-dependent drug absorption in the airway. Am J Respir Cell Mol Biol 2007;36(1):53-60

19. Luk CK, Dulfano MJ. Effect of $\mathrm{pH}$, viscosity and ionic-strength changes on ciliary beating frequency of human bronchial explants. Clin Sci (Sci) 1983;64(4):449-451.

20. ECRI Institute. ECRI Institute warns of equipment risks associated with use of inhaled epoprostenol [ECRI Exclusive Hazard Report]. March 24, 2016. Plymouth Meeting, Pennsylvania: ECRI Institute.
21. Davis MD, Suzaki I, Kawano S, Komiya K, Cai Q, Oh Y, et al. Tissue factor facilitates wound healing in human airway epithelial cells. Chest 2019;155(3):534-539.

22. Fulcher ML, Randell SH. Human nasal and tracheo-bronchial respiratory epithelial cell culture. Methods Mol Biol 2013;945:109-121.

23. Chan FK, Moriwaki K, De Rosa MJ. Detection of necrosis by release of lactate dehydrogenase activity. Methods Mol Biol 2013;979:65-70.

24. Martin TR, Hagimoto N, Nakamura M, Matute-Bello G. Apoptosis and epithelial injury in the lungs. Proc Am Thorac Soc 2005;2(3):214-220.

25. Carvalho TC, McConville JT. The function and performance of aqueous aerosol devices for inhalation therapy. J Pharm Pharmacol 2016; 68(5):556-578.

26. Sood BG, Peterson J, Malian M, Galli R, Geisor-Walter M, McKinnon $\mathrm{J}$, et al. Jet nebulization of prostaglandin E1 during neonatal mechanical ventilation: stability, emitted dose and aerosol particle size. Pharmacol Res 2007;56(6):531-541.

27. Pickering H, Pitcairn GR, Hirst PH, Bacon PR, Newman SP, Affrime MB, Marino M. Regional lung deposition of a technetium 99m-labeled formulation of mometasone furoate administered by hydrofluoroalkane 227 metered-dose inhaler. Clin Ther 2000;22(12):1483-1493.

28. Zanen P, Go LT, Lammers JW. The efficacy of a low-dose, monodisperse parasympathicolytic aerosol compared with a standard aerosol from a metered-dose inhaler. Eur J Clin Pharmacol 1998;54(1):27-30.

29. Zeman KL, Wu J, Bennett WD. Targeting aerosolized drugs to the conducting airways using very large particles and extremely slow inhalations. J Aerosol Med Pulm Drug Deliv 2010;23(6):363-369.

30. Stahlhofen W, Gebhart J, Heyder J, Philipson K, Camner P. Intercomparison of regional deposition of aerosol particles in the human respiratory tract and their long-term elimination. Exp Lung Res 1981;2(2):131-139.

31. Brown AT, Gillespie JV, Miquel-Verges F, Holmes K, Ravekes W, Spevak $\mathrm{P}$, et al. Inhaled epoprostenol therapy for pulmonary hypertension: Improves oxygenation index more consistently in neonates than in older children. Pulm Circ 2012;2(1):61-66.

32. Buckley MS, Feldman JP. Inhaled epoprostenol for the treatment of pulmonary arterial hypertension in critically ill adults. Pharmacotherapy 2010;30(7):728-740.

33. Davis MD, Walsh BK, Dwyer ST, Combs C, Vehse N, Paget-Brown A, et al. Safety of an alkalinizing buffer designed for inhaled medications in humans. Respir Care 2013;58(7):1226-1232.

34. Pilcer G, Amighi K. Formulation strategy and use of excipients in pulmonary drug delivery. Int J Pharm 2010;392(1-2):1-19.

35. Fan EKY, Fan J. Regulation of alveolar macrophage death in acute lung inflammation. Respir Res 2018;19(1):50.

36. Dahlem P, van Aalderen WM, de Neef M, Dijkgraaf MG, Bos AP. Randomized controlled trial of aerosolized prostacyclin therapy in children with acute lung injury. Crit Care Med 2004;32(4):1055-1060.

37. Searcy RJ, Morales JR, Ferreira JA, Johnson DW. The role of inhaled prostacyclin in treating acute respiratory distress syndrome. Ther Adv Respir Dis 2015;9(6):302-312.

38. Vane JR, Botting RM. Pharmacodynamic profile of prostacyclin. Am J Cardiol 1995; 75(3):3A-10A.

This article is approved for Continuing Respiratory Care Education credit. For information and to obtain your CRCE

(free to AARC members) visit

www.rcjournal.com

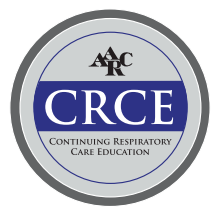

